\title{
EL DESARROLLO DE LAS COMPETENCIAS PROFESIONALES EN LA EDUCACIÓN UNIVERSITARIA
}

\author{
Alida Nersa Paneque Ginarte. \\ Profesora Carrera de Ingeniería Electromecánica. \\ Universidad Autónoma "René Gabriel Moreno". Santa Cruz de la Sierra. Bolivia. \\ apanequeginarte@gmail.com
}

\section{RESUMEN}

El artículo aborda la evolución que ha tenido la categoría competencia, desde lo laboral hasta llegar a lo educativo. En particular se ofrece la contextualización de la definición de competencias profesionales para la formación universitaria. Se analizan las tendencias en el desarrollo de competencias y su relación con los estándares para su valoración en el Modelo de formación por competencias.

Palabras clave: competencias y formación por competencias

\section{ABSTRACT}

The article discusses the evolution of competencies, from the workplace to the educational area. Particularly, it offers a contextualization of the definition of professional competencies for university education. It analyzes trends in the development of competencies, as well as their relationship with evaluation standards within the competency-based education Model.

Keywords: competencies - competency-based education

Recibido: Agosto 2013

Aprobado: Septiembre 2013

\section{Introducción}

El concepto de "competencia", aplicado al ambiente de trabajo, nace en la década de los setenta, y surge en un primer momento como competencia laboral. Este enfoque respondía a las necesidades de una época, donde existía una gran obsesión por la producción, dejando de lado la satisfacción y desarrollo del trabajador.

El concepto competencia laboral es cambiado por un nuevo enfoque, por el de Competencia Profesional.(1) Este último, rescata la integridad del ser humano que desarrolla determinada actividad productiva y en consecuencia reorienta el rumbo de la educación institucionalizada.

A partir de la década de los ochenta, los países industrializados han dado un gran impulso a la educación y a la capacitación basada en competencias, con resultados exitosos principalmente en Australia, Canadá, Francia, Estados Unidos de Norteamérica y el Reino Unido de la Gran Bretaña. (2)

Este concepto de competencias involucra dos elementos importantes: educación y capacitación. El primer elemento se ubica en las instituciones formadoras y el segundo en el ámbito laboral. Este movimiento ha dado origen a un nuevo pensamiento educativo totalmente opuesto al pensamiento tradicional en el cual existe una fuerte dicotomía entre educación académica y educación técnica, en otras palabras entre la teoría y la práctica. El presente artículo centra su atención en el desarrollo de las competencias como parte del perfil del egresado de la educación universitaria, desde una posición más integradora y humana.

\section{Desarrollo:}

Se comienza realizando una sistematización de las diferentes acepciones al término competencia para con posterioridad cerrar la intención investigativa a su acepción de competencias profesionales. Mal- 
pica, en el año 1996, señala que la relación entre las condiciones y demandas de las situaciones concretas en el trabajo (la práctica) con las necesidades de sistematización del conocimiento (la teoría), es más significativa para el individuo si la teoría cobra sentido a partir de la práctica; es decir, si los conocimientos teóricos se abordan en función de las condiciones concretas del trabajo y si se pueden identificar con situaciones originales.

Pero ésta no es una tarea fácil y requiere de una adecuada formación pedagógica y capacitación dentro de un modelo por competencias que permita a las personas involucradas en el PEA (Proceso de Enseñanza Aprendizaje), acortar la brecha existente entre la teoría y la práctica. Para ello, es necesario haber identificado las competencias que el mercado laboral requiere de los profesionales, y en base a ello diseñar programas de estudio adecuados que contemplen los requerimientos que demanda la sociedad de hoy.

El estudio de las competencias ocupa hoy un espacio de primer orden, y es tratado por diversas instituciones y autores, para reconocer determinadas tendencias en su tratamiento y definición.(3)

Las competencias constituyen, en la actualidad, una conceptuación y un modo de trabajo, en la gestión de los recursos humanos, que permiten una mejor articulación entre gestión, trabajo y educación.

El desarrollo del capital humano, repercute en el aumento de la capacidad de la producción, alcanzada con mejoras en las condiciones de trabajo y capacidades de los trabajadores. Estas capacidades se adquieren con el entrenamiento, la educación y la experiencia laboral, referidas al conocimiento práctico, las habilidades adquiridas y los conceptos aprendidos de un individuo, que lo hacen más competente. (4)

La definición de las competencias de las profesiones es una necesidad, tanto desde el punto de vista de su utilidad docente, de planificación y gestión de los servicios, como de la regulación del derecho al ejercicio de la profesión. (5)

En las dos últimas décadas se ha insistido en la necesidad de vincular la formación profesional con las necesidades de desempeño, en el área laboral, de ahí que los modelos educativos basados en competencias laborales o profesionales se hayan difundido en las instituciones educativas a nivel mundial.(6) Esta tendencia internacional involucra toda el área técnica, educación media superior y también la formación universitaria.

La formación de los profesionales de las ciencias técnicas, a partir de las competencias, permite es- tablecer un vínculo entre la teoría y la práctica, lo que conlleva a una integración de los conocimientos y las habilidades asociadas al aprendizaje, que promueven actitudes y disposiciones para pensar y actuar de forma integral, cumpliéndose así con el encargo social, en el caso del presente artículo, formar profesionales capaces de actuar competentemente; sin embargo, no se hace explícita la presencia de los valores morales, tan importantes para un profesional.

La competencia es un tipo de enseñanza, único y exclusivo, de los seres humanos, que involucra las tres dimensiones de la mente humana: el saber y el hacer (dimensiones cognitivas) y la dimensión afectiva (cognitivo expresiva) que son las actitudes, sentimientos y valores. Por ello, el desarrollo de competencias debe ir más allá de transmitir saberes y destrezas manuales, debe buscar incrementar la capacidad de las personas y los aspectos culturales, sociales y actitudinales.

El término competencias no es un ejercicio simple, la misma conlleva valores tales como la concepción del modo de producción y transmisión de conocimientos, sobre la relación educación - sociedad, de la misión y valores del sistema educativo, de la práctica de enseñanza y de evaluación de los docentes, y las actividades y desempeño de los estudiantes.

De forma amplia se puede definir como las "capacidades que todo ser humano necesita para resolver, de manera eficaz y autónoma las situaciones de la vida",(7) se fundamentan, en un saber profundo, no sólo en saber qué y cómo, sino ser persona en un mundo complejo, cambiante y competitivo.

Acercamiento teórico al concepto de Competencias Profesionales.

En el concepto de competencia no existe una definición única, ya que este término tiene múltiples acepciones, y en ocasiones nominaciones diferentes en el contexto educativo. Las tres acepciones más difundidas son: capacidad, que es el saber hacer con los conocimientos adquiridos, competitividad que hace alusión a una sociedad en la que se tiene que demostrar ser el mejor o el más eficiente, e incumbencia que es la función que debe ser desempeñada por una persona o aquello que involucra afectivamente a un individuo. (8)

Existen varias definiciones, correspondientes a enfoques distintos, una de las más aceptadas es la concepción de "competencia como relación holística o integrada", en la cual toda competencia 
se expresa como un complejo estructurado de atributos generales (conocimientos, habilidades, actitudes y valores) requeridos para interpretar situaciones específicas y desempeñarse en ellas de manera científica.

Bajo un enfoque holístico o integrado, dicha concepción, reúne tanto las habilidades derivadas de la combinación de atributos, como las tareas determinadas para situaciones específicas, y de esta forma, tomar en cuenta, el contexto y la cultura del lugar de trabajo en el que se genera el proceso. De acuerdo con esta noción integradora, las competencias involucran no sólo los conocimientos y técnicas, sino que además comprometen la ética y los valores, como elementos de desempeño competente, la importancia del contexto y la posibilidad de demostrarlo de diferentes maneras. (9)

Se puede definir competencia, como un proceso dinámico y longitudinal en el tiempo, por el cual una persona utiliza los conocimientos, habilidades, actitudes y buen juicio, asociados a su profesión, con la finalidad de poder desarrollarla de forma eficaz en todas las situaciones que corresponden al campo de su práctica. Representa, por tanto, un estado de su praxis profesional.(10) La importancia o el peso relativo de cada una de éstas, dependerá obviamente de la profesión e incluso de la especialización de cada profesión. (11)

Otros autores las definen como, complejas capacidades integradas en diversos grados, que la educación debe formar en los individuos para que pueda desempeñarse como sujeto responsable en diferentes situaciones de la vida social y personal, y de esta forma: saber, ver, hacer, actuar y disfrutar convenientemente, (12) evaluar alternativas, elegir las estrategias adecuadas y hacerse cargo de las decisiones tomadas.

Este desempeño responsable de los individuos, según expresó el Dr. Tobón en el año 2006, va más allá de analizar las consecuencias, antes de actuar, para corregir los errores lo más pronto posible, sino que en las competencias, toda actuación es un ejercicio ético, en tanto se hace necesario predecir las consecuencias del desempeño, revisar cómo se ha actuado y corregir los errores de las actuaciones, lo cual incluye reparar posibles perjuicios a otras personas o a sí mismo.

Mertens, en el año 2000, define que la competencia laboral es la aptitud de un individuo para desempeñar una misma función productiva en diferentes contextos y con base a los requerimientos de calidad esperados por el sector productivo. Esta aptitud se logra con la adquisición y desarrollo de conocimientos, habilidades y capacidades que son expresados en el saber, el hacer y el saber hacer. (13)

Según Robert Norton, competencia son los "conocimientos, actitudes y destrezas necesarias para desempeñar una ocupación dada". (14) Por su parte, Zúñiga Vargas definió competencia como un "conjunto de elementos que revisten un significado claro en el proceso del trabajo y su ejercicio; las funciones del profesional; requerimientos relacionados con la salud, seguridad, calidad y relaciones en su puesto de trabajo". (15)

La competencia laboral pretende ser un enfoque integral de formación que desde su mismo diseño, conecta el mundo del trabajo y la sociedad en general, con el mundo de la educación. (16) En América Latina, países como México, Brasil, Argentina, Costa Rica y Chile, entre otros, ejecutan proyectos de certificación de competencias, para el medio laboral y sistemas de formación, que apuntan a mejorar la certificación; así como, a incorporar el enfoque de competencia laboral con fines de actualización de los programas de formación. (17)

En las dos últimas décadas se ha insistido en la necesidad de vincular la formación profesional con las necesidades de desempeño en el área laboral, de ahí que los modelos educativos basados en competencias laborales o profesionales se hayan difundido en las instituciones educativas a nivel mundial. En la actualidad resulta necesario, encontrar procedimientos para formar profesionales competentes. Sin embargo, la educación basada en competencias ha sido objeto de múltiples interpretaciones.

Kane; define la Competencia Profesional como "el grado de utilización de los conocimientos, las habilidades y el buen juicio asociados a la profesión, en todas las situaciones que se pueden confrontar en el ejercicio de la práctica profesional". (18)

En esta definición, que es una de las más dinámicas y completas; por una parte, se relaciona a la competencia con la aplicación de los conocimientos, habilidades y razonamientos, de la realidad del profesional y por otra, se encuentra una dimensión que es fundamental: la capacidad de un profesional de utilizar su buen juicio, o sea, de razonar para tomar decisiones.

La Competencia Profesional como capacidad de desempeño, depende de tres componentes: el perfil profesional determinado por las aptitudes y rasgos de personalidad, los conocimientos, habilidades y actitudes adquiridas en la formación, y las desarrolladas en la práctica profesional. Esto 
implica conocimientos y acciones unidas a las actitudes y valores personales.

Las Competencias Profesionales definen el ejercicio eficaz de las capacidades que permiten el desempeño de una ocupación, respecto a los niveles requeridos en el empleo. "Es algo más que el conocimiento técnico que hace referencia al saber y al saber - hacer". El concepto de competencia engloba no sólo las capacidades requeridas para el ejercicio de una actividad profesional, sino también un conjunto de comportamientos, facultad de análisis, toma de decisiones, transmisión de información, etc., considerados necesarios para el pleno desempeño de la ocupación. (19)

Urbina Laza Omayda la define como, "la integración de conocimientos, habilidades, conductas, actitudes, aptitudes y motivaciones conducentes a un desempeño adecuado y oportuno en diversos contextos". (20)

La competencia laboral es la construcción social de aprendizajes significativos y útiles para el desempeño productivo en una situación real de trabajo que se obtiene no sólo a través de la instrucción, sino también y en gran medida mediante el aprendizaje por experiencia en situaciones concretas de trabajo.

Desde el análisis documental, se reconoce a la OIT (Organización Internacional del Trabajo) como una de las organizaciones que ha definido el concepto de Competencia Profesional "como la idoneidad para realizar una tarea o desempeñar un puesto de trabajo eficazmente por poseer las calificaciones requeridas para ello". (21) En este caso, los conceptos competencia y calificación, se asocian fuertemente dado que la calificación se considera una capacidad adquirida para realizar un trabajo o desempeñar un puesto de trabajo, en muchos casos incorporando este sistema de Competencias Profesionales como calificador de cargos y funciones de las diferentes ocupaciones o áreas profesionales y/o técnicas.

Un profesional puede ser competente, pero ante una situación real no demostrar el saber hacer y el buen juicio, por lo que no responde al nivel profesional de calidad exigible en aquella situación.

En este sentido, las competencias no se pueden reducir al desempeño laboral, tampoco a la sola apropiación de conocimientos para saber hacer, sino que en ella está implicado todo un conjunto de capacidades, que se desarrollan a través de procesos, que conducen a la persona responsable a ser competente para realizar múltiples acciones por las cuales, proyecta y evidencia su capacidad de resolver un problema dado, dentro de un contexto especifico y cambiante. Tampoco se puede reducir las competencias a los modos de actuación de las personas exclusivamente, en ellas se origina un sistema multidimensionado que expresa las relaciones entre individuo, contexto laboral y toma de decisiones en correspondencia con el momento histórico - concreto que le ha tocado vivir a cada obrero, técnico o profesional.

Por su naturaleza y a partir de su concepción original, por la forma en que se adquieren o desarrollan, las competencias se clasifican en: académicas, laborales y profesionales. Cada una contiene capacidades agrupadas en tres niveles: esencial o básico, genérico y específico. Existiendo una relación de interdependencia entre ellas. Existen otros investigadores que reconocen esta agrupación de los niveles de desarrollo de las competencias en función de la formación que se trate, en tal sentido se reconoce un nivel básico y otro especializado.

Así la formación integral se va desarrollando por niveles de complejidad en los diferentes tipos de: "Competencias básicas o fundamentales; genéricas o comunes; específicas o especializadas y laborales". (22)

Esta clasificación de las competencias obedece al comportamiento externo del sujeto en su desempeño, refleja los saberes (sólo conocimientos) de estos profesionales en los servicios que atiende, pero no incluye la posición ética del sujeto ante su desempeño y responsabilidad social.

Todas estas definiciones son una muestra de la multiplicidad de definiciones que existen al respecto, pero la autora las considera suficientes para llegar a una conclusión, que la definición no es definitiva, su dinámica cambia en correspondencia con el contexto laboral, la formación adquirida con anterioridad y el sistema de relaciones que establece el sujeto durante su desempeño profesional y/o técnico, elementos de gran valor para el presente artículo.

De estas definiciones presentadas, se puede inferir que una competencia integra un conjunto de conocimientos, habilidades y comportamientos del individuo para desempeñar con éxito una actividad dada. Dicho de otro modo, una competencia integra los cuatro pilares de la Educación para el Siglo XXI: saber, saber hacer, saber ser y saber actuar.

Ser Competente: definición y naturaleza del concepto. 
En un principio, el desarrollo de competencias laborales tenía una fuerte inclinación hacia lo normativo. Lo que en realidad se proponía era establecer ciertas normas que un individuo debía cumplir para ser considerado competente en el campo laboral. Todo esto se relacionaba solamente con la práctica y dejaba de lado otros atributos, tales como, los valores y actitudes que todo profesional debía poseer a la hora de ejercer una determinada profesión.

Sin embargo, ser competente significa mucho más que eso. Es de suma importancia resaltar que, competente es aquella persona que domina o posee un conjunto de atributos (conocimientos, habilidades, actitudes y valores) necesarios para el desempeño de un trabajo o actividad, es decir, los utiliza en combinaciones diferentes para desempeñar distintas tareas ocupacionales. Vale decir que, una vez que un sujeto ha desarrollado una competencia, ésta puede ser observada en otro contexto, y hasta, quizás en situaciones más complejas.

De acuerdo a esta última idea, se puede afirmar que el concepto de competencia es correlativo ya que vincula diferentes elementos, atributos y tareas dentro de una estructura conceptual, mientras que el pensamiento tradicional sólo se concentra en las tareas que se necesitan desempeñar, o bien en los atributos genéricos o las capacidades que sostienen la competencia sin tomar en cuenta la forma en que estas necesidades se aplican a diferentes contextos.

Por otro lado, es importante destacar el carácter holístico del concepto de competencia ya que toma en cuenta la cultura y el contexto de trabajo. Desde esta óptica, se plantea que la formación impulsada por una institución educativa (en este caso la universidad) no sólo debe diseñarse en función de la incorporación del sujeto a la vida productiva a través del empleo, sino más bien, "partir de una formación profesional que además de promover el desarrollo de ciertos atributos (conocimientos, habilidades, actitudes, aptitudes y valores), considere la ocurrencia de varias tareas (acciones intencionales) que suceden simultáneamente dentro del contexto (y la cultura del lugar de trabajo) en el cual tiene lugar la acción; y a la vez permita que algunos de estos actos intencionales sean generalizables". (23)

En otras palabras, el individuo al comprender progresivamente la cultura de sus ocupaciones y el contexto en el cual se desenvuelve, será capaz de integrar no sólo su conocimiento técnico, sino también, podrá desarrollar habilidades, actitudes y a la vez tomar decisiones individuales y/o gru- pales en una situación determinada, en la cual se encuentre involucrado.

Finalmente, la sistematización realizada a los conceptos de competencia y competente tienen una dimensión psicológica cognoscitiva, la cual enfatiza la importancia de la experiencia a través del aprendizaje en el lugar de trabajo, centrando la atención en el concepto de "aprender haciendo". Este enfoque de aprendizaje promueve la experiencia individual, presentándole al individuo situaciones, problemas o actividades que debe resolver o analizar por sí mismo o por el grupo. En tal sentido, la autora seguidamente centra la atención en cómo organizar el desarrollo de las competencias.

El proceso de organización del desarrollo de competencias.

El estudio histórico - lógico realizado alrededor del proceso de organización de la formación y desarrollo de las competencias en general, ha permitido a la autora conocer que para organizar sistemas o procesos de desarrollo de competencias, se deben considerar un conjunto de condiciones que garantizan un resultado favorable a la formación, éstas son las siguientes:

1. Las competencias deben ser identificadas a partir de un proceso de diagnóstico.

$2 . \quad$ Las competencias que se quieren desarrollar deben manifestar sus relaciones con los objetos de la profesión o el desempeño.

$3 . \quad$ Las competencias que se desean modelar deben verificarse ante especialistas de las instituciones empleadoras, expertos del área de conocimientos o consensuadas con los propios estudiantes que serán formados y que cuentan con las vivencias que la práctica cotidiana les aporta.

4. Cada competencia modelada debe identificar las formas de evaluación que permitirá constatar el grado de adquisición de forma permanente y sistémica, en su relación con los servicios que realiza y en correspondencia con el resto de las que ya posee o que se le están formando simultáneamente.

5. La evaluación de las competencias debe tomar en consideración los conocimientos, las actitudes, los valores propios del desempeño y los modos de actuación que en el desempeño requiere el sujeto, como las principales fuentes de evidencia y criterios para la medición.

6. El desarrollo de competencias es un proceso de aprendizaje personalizado e individualizado, en tanto que no debe estar sujeto a rígidas estrategias o dosificaciones que avancen el programa sin la constancia de que ya poseen los conocimientos, habilidades, valores, actitudes y 
modos de actuación necesarios para continuar la formación hacia un nivel mayor de desarrollo.

7. El desarrollo de competencias requiere de una retroalimentación constante del docente que lo lleva a cabo, los materiales principales empleados como medios de enseñanza deben ser aquellos que reflejen situaciones reales y experiencias en el trabajo en sus diferentes objetos o áreas de desempeño.

8. El desarrollo de competencias debe estar dirigido, en su mayoría, a la adquisición de experiencias prácticas, sin olvidar los temas o áreas del conocimiento que fundamentan sus acciones. Éstas pueden estar relacionadas con hechos, conceptos, principios y otros tipos de conocimientos, deben ser parte integrante de las tareas y funciones propias del puesto de trabajo o cargo que ocuparán (Perfil ocupacional).

En los últimos tiempos se ha podido apreciar una tendencia a identificar como elementos de competencias:

- Las capacidades, habilidades o destrezas en los modos de actuación de los sujetos.

- Las funciones propias de los objetos de trabajo que atiende.

- Los modos de actuación de los recursos laborales en su desempeño.

- Las prioridades de los centros o instituciones donde laboran.

En casi todos estos elementos estructurales, si bien es cierto que en el orden metodológico y práctico, ofrece una guía para su conformación, las competencias quedan fuera del sujeto que se forma, sea desde el puesto de trabajo o no, dejando marcadamente el carácter estructuralista o funcionalista en el plano epistemológico.

Es por ello que, desde hace una década la preocupación ha sido por valorar los niveles de profesionalización desde el desempeño y desde la formación por competencias; abordando los elementos inter-subjetivos de los sujetos, más a tono con las concepciones humanistas, que los lleve a la concientización del desarrollo de los niveles de profesionalización y desempeño; que ofrezca, no sólo, el cumplimiento de las responsabilidades sociales, laborales, estudiantiles o profesionales; sino además, que permita la satisfacción de la persona por lo que hace y cómo lo hace.

Otra de las tendencias, en el desarrollo de competencias, está asociada con los estándares para su valoración; en tal sentido, se puede estudiar que los más comunes y adaptables a diferentes profesiones, oficios y contextos laborales y educativos, son los Niveles de Asimilación identificado por el carácter en que se apropian del conocimiento, las habilidades y valores, éstos son los Niveles de Reproducción, Aplicación y Creación, según expresa en su tesis Pérez Hernández R., en el año 2006.

La autora, desde la sistematización realizada, coincide con el carácter sumativo de las habilidades y su posición como habilidad, operación o destreza depende, de la utilización que se le dé en la práctica educativa.

El nivel de Reproducción, permite dominar la comprensión consciente del movimiento del conocimiento de un objeto determinado. Aquí se busca lo esencial o más importante de ese hecho, concepto o ley, objeto de conocimiento, fijado en la memoria. Constituye el grado o nivel de desarrollo del saber, conocimientos y habilidades intelectuales o teóricas. Este grado se logra a través de distintas habilidades, tales como: describir, explicar, identificar, clasificar, determinar, dosificar, comparar, valorar, concretar, entre otras.

El nivel de Aplicación, se caracteriza por la posibilidad de utilizar eficientemente los conocimientos y habilidades en la solución de ciertos problemas y situaciones nuevas.

En este nivel se desarrolla el grado del Saber Hacer (Teórico - Práctica), donde se manifiestan con más fuerza las habilidades: clasificar, comparar, concretar y valorar. Cuando la aplicación de estos conocimientos y habilidades se realiza en una situación conocida, entonces será del nivel de asimilación de reproducción.

El nivel de Creación presupone la capacidad de adquirir conocimientos y habilidades con el hábito de investigar e innovar. El grado de desarrollo es "hacer" con conocimientos y habilidades prácticas. Se desarrollan como habilidades: operar, elaborar, reparar, innovar, investigar, entre otras.

Los especialistas de diseño curricular, en la actualidad y animados por el enfoque histórico - cultural, establecen un conjunto de elementos - llámense etapas, fases, áreas, entre otras acepciones - que debe disponer todo Modelo de formación por competencias, entre éstos, se encuentran:

1. Diagnóstico de los problemas que generan la necesidad de la formación por competencias.

2. Mapa de competencias. En este componente se deben identificar:

- Las competencias en sus diferentes tipos (básicas o especializadas; prácticas o teóricas; generales o específicas, otras).

- El sistema de conocimientos de cada com- 
petencia o de todas como un sistema de competencias, que es más conveniente en los currículos, en tanto que evitan repeticiones innecesarias.

- El sistema de habilidades y/o capacidades propias de los objetos de la profesión u ocupación que se relacionan en el perfil de egresado (parte integrante del diseño de planes de estudios o programas).

- El sistema de valores asociados a cada competencia o sistema de competencias que revelan el carácter educativo del modelo y por ende su carácter formativo o actitudinal.

\section{Conclusión}

1.- Los profesionales de la educación debe estar al tanto de las diferentes etapas en el aprendizaje del estudiante y la forma como él puede ayudar en su formación integral, al crear ambientes para potenciar y desarrollar las diferentes habilidades, destrezas y valores necesarios en el nuevo profesional que requiere la sociedad en su conjunto y el mundo del trabajo en particular a partir de las competencias profesionales que se identifican en el perfil del egresado y que responden a las exigencias del mercado laboral.

\section{Referencias bibliográficas:}

1.- Andrew Gonczi R. Enfoque de la educación basada en competencias: segunda parte; 2010.

2.- Añorga J., Valcárcel N. y Pérez García A. Competencias profesionales. Instituto Superior Pedagógico "Enrique José Varona". La Habana, Cuba; 2008.

3.- Guedes V. El conocimiento y las competencias en las organizaciones del siglo XXI. En: Universidad Metropolitana. Caracas, Venezuela: Epsilon Libros; 2002.

4.- Spencer JR., Mc Clelland D., Spencer M. Competency Assessment Methods: history and State of Art. Hay $\urcorner$ Mc Research Pres; 1994. (consultado: febrero de 2010).

5.- Paravic T. Postgrado stricto sensus del departamento de enfermería de la Universidad de Concepción. Concepción, Chile; 2004.

6.- Larios Mendoza H. El ejercicio actual de la medicina. Competencia profesional y competencia clínica. La Habana, Cuba; 2006.

7.- Ibídem 1.

8.- Montalvo Perdomo S. Propuesta de Compe- tencias y Funciones para el futuro especialista en Enfermería Clínico Quirúrgica en Cuba. (Tesis en Opción al título de Máster en Enfermería). La Habana, Cuba; 2008.

9.- Moreno R. La educación basada en normas de competencia como un nuevo modelo de formación profesional en México. En: Pensamiento Universitario 91, tercera época. Centro de Estudios sobre la Universidad. México; 2005.

10.- Reflexiones y Perspectivas de la Educación Superior en América Latina. Informe Final. Proyecto Tuning - América Latina; 2007.

11.- Algunos aportes al concepto de competencias desde la perspectiva de América Latina. Buenos Aires, Argentina; 2004.

12.- Cullen C. El debate epistemológico de fin de siglo y su incidencia en la determinación de competencias, en los diferentes niveles de educación formal. En: Novedades Educativas; 1996. Buenos Aires, Argentina.

13.- Mertens L. La gestión por competencia laboral en la empresa y la formación profesional. Madrid, España: Organización de Estados Iberoamericanos para la Educación, la Ciencia y la Cultura (OEI); 2000.

\section{4.- Ibídem 2.}

15.- Zúñiga Vargas F. Competencias claves y aprendizaje permanente. Montevideo, Uruguay: CINTERFOR; 2000.

16.- Irigoin M. y Vargas F. Competencia laboral: manual de conceptos, métodos y aplicaciones en el sector salud. Montevideo, Uruguay: CINTERFOR; 2002.

17.- Ibídem 39.

Ibídem 2.

18.- IBERFOP-OEI. Programa Iberoamericano para el diseño de la formación profesional, Conceptos básicos de competencias laborales. CINTER/OIT, Madrid, España; 1998.

19.- Torres Esperón M. y Urbina Laza O. Funciones y Competencias en Enfermería. La Habana, Cuba: Editorial Ciencias Médicas; 2008.

20.- Centro Interamericano de Investigación y Documentación sobre Formación Profesional, de la Organización Internacional del Trabajo. (OIT/ CINTERFOR). Las 40 preguntas más frecuentes sobre formación por competencias. Disponible en 
URL: http://www.oitcinterfor.org/public/spanish/region/ampro/cinterfor/index.htm. Consultado: enero $20,2011$.

21.- Ibídem 11.

22.- Ibídem 2.

23.- Ibídem 20

\section{Bibliografía}

1. Añorga J. y otros. La producción intelectual: proceso organizado y pedagógico. La Habana, Cuba: Editorial UH; 2003.

2. Añorga J., Valcárcel N., Pérez García M. Competencias profesionales. Instituto Superior Pedagógico "Enrique José Varona". La Habana, Cuba; 2008.

3. Cullen C. El debate epistemológico de fin de siglo y su incidencia en la determinación de competencias, en los diferentes niveles de educación formal. En: Novedades Educativas; 1996. Buenos Aires, Argentina.

4. Forgas J. Diseño curricular por competencias: una alternativa para la formación de un técnico competente. Curso 14, Pedagogía. La Habana, Cuba; 2003.

5. García del Portal J. Sobre la enseñanza de la Ingeniería. El Informe Technion. Revista Cubana de Educación Superior. 1990, Vol. X, No.1. La Habana, Cuba.

6. Guedes V. El conocimiento y las competencias en las organizaciones del siglo XXI. En: Universidad Metropolitana. Caracas, Venezuela: Epsilon Libros; 2002.

7. Malpica José. Los modelos de gestión por competencias. España: Editorial Reverté; 1996.

8. Mertens Leonard. Seminario Taller Competencias Laborales. Metodología AMOD para la construcción de Currículum de Capacitación. Buenos Aires, Argentina; 1998.

9. Paneque G. A y Valcárcel N. Aproximaciones a la formación por competencias, una vía para acercar el micro al macrocurrículo en una carrera universitaria. En: Revista Órbita Científica. 2010. La Habana, Cuba; p.2.

10. Pérez Hernández R. Modelo del perfil de los cargos por competencias laborales del técnico medio en construcción civil para el mejoramiento de la formación y la eficiencia del trabajo. (Tesis doctoral). La Habana, Cuba: Instituto Superior Pedagógico "Enrique José Varona”; 2006.
11. Tobón S. Formación basada en competencias. Pensamiento complejo, diseño curricular y didáctica. Bogotá, Colombia: Ediciones Ecoe; 2004.

12. Tobón S. Aspectos básicos de la formación basada en competencias. Proyecto Mesesup. Talca, Chile; 2006.

13. Torres Esperón M., Urbina Laza O. Funciones y Competencias en Enfermería. La Habana, Cuba: Editorial Ciencias Médicas; 2008.

14. Zúñiga Vargas F. Competencias claves y aprendizaje permanente. Montevideo, Uruguay: CINTERFOR; 2000. 